\title{
Reorganización logística y espacial de las cadenas de suministro marítimo-portuarias en México: entre el neoproteccionismo comercial y la pandemia del COVID-19

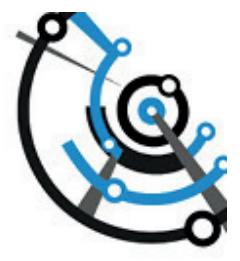

(4) Carlos Daniel Martner Peyrelongue

Instituto Mexicano del Transporte, México.

ORCID: https://orcid.org/oooo-0002-7676-4256

Recibido: 26 de abril de 2021. Aceptado: 24 de junio de 2021.

\begin{abstract}
Resumen
En los últimos años, las cadenas de suministro marítimo-portuarias movilizadas a través de redes globales de transporte intermodal han enfrentado escenarios adversos derivados tanto de las tensiones geopolíticas expresadas en el neoproteccionismo y la guerra comercial entre las principales potencias mundiales, como en la aparición fulminante y demoledora de la pandemia del COVID-19.
\end{abstract}

En este contexto, han quedado evidenciadas ciertas vulnerabilidades de la fragmentación logística y espacial de los procesos productivos derivados de la globalización económica que ha primado en las últimas tres décadas. La guerra comercial entre países centrales de la economía-mundo, pero sobretodo la irrupción de la pandemia del COVID-19 provocaron desajustes enormes que interrumpieron de manera abrupta la regularidad y continuidad del suministro de insumos y bienes de las cadenas productivas extensas que se articulan mayoritariamente en un espacio global a través de complejas redes de transporte marítimo e intermodal.

Derivado de lo anterior, la presente propuesta busca avanzar en la identificación de los impactos logísticos y espaciales del COVID-19 en el transporte marítimo de mercancías y en los puertos mexicanos, así como visualizar, a grandes rasgos, las tendencias emergentes de articulación espacial de las cadenas de suministro globalizadas, con la finalidad de aportar elementos cualitativos que eventualmente contribuyan o coadyuven a la construcción de escenarios post-pandemia, a partir de un enfoque teóricometodológico de tipo estructural-sistémico, basado en la conformación de un espacio de redes y flujos globales. 


\title{
Logistic and spatial reorganization of maritime-port supply chains in Mexico: Between trade neo-protectionism and the COVID-19 pandemic
}

\begin{abstract}
In recent years, the maritime-port supply chains mobilized through global intermodal transport networks have faced adverse scenarios derived both from the geopolitical tensions expressed in neo-protectionism and the trade war between the main world powers, as well as the sudden and devastating appearance of the COVID-19 pandemic.

This context has provided evidence of certain vulnerabilities of the logistical and spatial fragmentation of the productive processes derived from the economic globalization that have prevailed in the last three decades. The trade war between central countries of the world-economy, but above all the irruption of the COVID-19 pandemic caused huge imbalances that abruptly interrupted the regularity and continuity of the supply of inputs and goods from the extensive productive chains that are mainly articulated in a global space through complex maritime and intermodal transport network.

Arising from the above, this proposal seeks to advance in the identification of the logistical and spatial impacts of COVID-19 in the maritime transport of goods and in the Mexican ports, as well as to visualize, broadly speaking, the emerging trends of spatial articulation of globalized supply chains, with the aim of provide qualitative elements that eventually contribute or assist to the construction of post-pandemic scenarios, based on a structural-systemic theoretical-methodological approach, based on the creation of a networks space and global flows.
\end{abstract}

Keywords: Maritime transport. Ports. Logistics. COVID-19. Neo-protectionism.

Palavras-chave: Transporte marítimo. Portas. Logística. COVID-19. Neoprotecionismo.

\section{Introducción}

Recientemente han sido cuestionados fuertemente los resultados del modelo económico predominante en las últimas tres décadas, basado, entre otras cosas, en la liberalización de la producción y las finanzas, en la privatización de los activos previamente manejados por el Estado, en la apertura comercial y en un proceso de hiperglobalización de las relaciones económicas, culturales y sociales (CEPAL, 2016; Fernández, 2018). El descontento deriva de la exclusión de vastos sectores de la sociedad y de muchas regiones del mundo de los beneficios de ese proceso globalizador que, en el discurso dominante, generaría bienestar y un mayor nivel de vida para la mayoría de la población mundial.

Desde la perspectiva económica, los conflictos sistémicos a raíz de los resultados de la globalización también se han acentuado, derivando en la paralización y/o revisión de muchos tratados comerciales (y en algunos casos la desanexión de países miembros), la imposición creciente de aranceles a los flujos internacionales de mercancías por parte de las grandes economías (principalmente, Estados Unidos), una guerra comercial en curso que, desde el año 2018, planteó muchas incógnitas sobre las nuevas reglas del juego de la economía-mundo y una posible reconfiguración espacial de las cadenas productivas que se encuentran altamente internacionalizadas.

A este complejo entorno se sumó, desde el primer trimestre del año 2020, los efectos de la pandemia global del COVID-19, que reveló múltiples fragilidades del modelo social, político y económico en prácticamente todas las naciones del planeta. En este contexto, 
han quedado evidenciadas también las limitaciones espaciales, logísticas y de distribución física de las redes productivas fragmentadas a escala global, expresadas en la interrupción diferenciada de las cadenas de suministro según la etapa de avance de la pandemia en cada continente y región del mundo, lo cual ha dificultado enormemente la producción de múltiples insumos y bienes y está planteando dificultades para la reactivación económica que se espera con ansias al superar las fases más difíciles del COVID-19.

Ante estas dificultades e inconvenientes derivados tanto del neoproteccionismo, como de la pandemia en curso, cabe plantear la hipótesis de una nueva regionalización de las actividades productivas en la que, entre otras cosas, se modificarán las cadenas de suministro vinculadas al transporte marítimo, a los puertos y a los corredores multimodales terrestres, donde eventualmente es factible asistir a un escenario de reconfiguración espacial en los litorales mexicanos y de su articulación con las regiones interiores.

En este sentido, la presente propuesta se apoya en un enfoque teórico-metodológico de tipo estructural-sistémico (Wallerstein, 1996, 2016) basado en la conformación de un espacio de redes y flujos de la globalización (Sassen, 2003; Castells, 2006, 2010; Dollfus, 2007; Veltz, 2006, 2014; Martner, 2010, 2020) y tiene por objetivo avanzar en la identificación de los impactos logísticos y territoriales del COVID-19 en el transporte marítimo de mercancías y en los puertos mexicanos, así como visualizar, a grandes rasgos, las tendencias emergentes de articulación espacial de las cadenas de suministro globalizadas, con la finalidad de aportar elementos cualitativos que eventualmente contribuyan o coadyuven a la construcción de escenarios Post-Covid19, en el contexto de los nuevos arreglos comerciales (el nuevo tratado norteamericano conocido como T-MEC, entre otros) y de las dificultades y vulnerabilidades para la movilidad de las mercancías y las personas impuestas por una suerte de neoproteccionismo regionalizado y por la propia dinámica de la pandemia.

\section{Globalización y conectividad espacial de los sistemas de transporte}

Una de las características fundamentales de la globalización, desde el punto de vista tecnológico y organizacional, ha sido la posibilidad de fragmentar los procesos productivos, permitiendo que cada fase de la fabricación de un producto se realice en territorios, regiones, países e incluso continentes distintos, en función de las ventajas competitivas que proporciona cada país, región o ciudad.

Esta transformación, provocó un desdoblamiento de la esfera de la producción sobre el territorio. En efecto, en el periodo previo a la globalización, el proceso productivo estaba básicamente confinado en un solo lugar (la fábrica), pero, en las últimas décadas se ha desdoblado sobre vastos espacios dispersos (donde se realiza cada una de las fases mencionadas), por lo tanto, la necesidad de mover flujos crecientes de bienes intermedios, es decir, en proceso de fabricación, implica una intensificación de la movilidad de las mercancías como nunca antes, una aceleración de la circulación física de los flujos materiales inédita en periodos previos (Martner, 2020).

Desde la perspectiva de la geografía (económica, humana, y de los transportes) los cambios son inconmensurables. Esto significa alteraciones notables en la movilidad, en la conectividad de los sistemas de transporte, en los territorios. Significa que ahora hay que realizar un gran número de traslados en el territorio para integrar el producto final. En otras palabras, significa más transporte. Por ello, se considera que la intensificación de la conectividad y de la movilidad es una característica consustancial a la globalización (Martner, 2020: 236). 
En este sentido, la globalización económica, junto con las recientes olas de innovación tecnológica, han propiciado la constitución de extensas redes que acercan fragmentos o nodos territoriales selectos dentro del proceso de conexión de la producción a escala global. Así, nunca antes en la historia se ha banalizado tanto la importancia de la distancia física entre los lugares. Hay una suerte de anulación de la distancia por el tiempo que permite que fragmentos territoriales distantes vinculados por redes globales de producción y circulación de pronto puedan encontrarse más integrados entre sí que con sus regiones contiguas.

Las relaciones de proximidad, de cohesión geográfica y cultural entre un centro o polo y sus áreas contiguas ahora tienden a desestructurarse por la emergencia de lo que Veltz $(1999,2014)$ ha llamado el territorio de redes, donde predominan las relaciones entre nodos fragmentados y territorialmente discontinuos que, sin embargo, se conectan o articulan, aún a largas distancias, con el apoyo de las nuevas tecnologías de transportes y comunicaciones.

La configuración de ese espacio global de redes y flujos de bienes tangibles e intangibles se convirtió en el sustento físico y tecnológico de la globalización en la presente fase de la economía-mundo capitalista. Desde luego, no todo es red, pues como señaló en su momento Santos (, 2000, p. 227) ..., vastas áreas escapan a ese diseño reticular, ..., son magmas o zonas de baja intensidad. Por tal razón, puede decirse que el territorio de redes es parcial y selectivo, pero sus efectos se dejan sentir de manera generalizada. Por ejemplo, la mayor parte de los puertos mexicanos, que en el periodo previo cumplían una función local, enfrentan dificultades para articularse a este espacio de redes y flujos de la globalización, lo cual ha propiciado prolongados procesos de estancamiento y declinación.

En esta misma perspectiva, se habla de un espacio reticular que preside una nueva forma de sociabilidad a distancia. Ese nuevo discurso del espacio de la conectividad y las redes

..., es el lenguaje de las normas y órdenes que actores lejanos hacen repercutir instantánea e imperativamente sobre otros lugares distantes. Tales redes constituyen los más eficaces transmisores del proceso de globalización al que asistimos (Santos, 2000: 225).

La intención última de las redes y sus nodos es activar flujos de diversa índole (capitaldinero, mercancías, órdenes de producción, valores, símbolos) que den continuidad al proceso de acumulación de capital y a la reproducción de la economía-mundo capitalista. Este es el sentido en el que Castells (2006) habla de la creación de un espacio de flujos que superpone y desestructura los vínculos territoriales previos, fundados en la proximidad y en rasgos comunes de identidad.

De esta manera, los imperativos de fluidez y aceleración de la circulación se convierten en un tema toral para darle viabilidad a la globalización.

Se establecen lugares y se crean objetos (como puertos, aeropuertos, terminales intermodales, autopistas, oleoductos, etc.) para favorecer la fluidez. En la actualidad, estos objetos transmiten valor a las actividades que los utilizan. En este caso podemos decir que circulan. Es como si ellos también fuesen flujos (Santos, 2000: 232).

No cabe duda que, con la fragmentación de los procesos productivos a escala global, la circulación ha adquirido un protagonismo inédito. Los nuevos métodos de producir, basados en la logística, es decir, en el manejo estratégico de los flujos materiales e inmateriales, en la información de la demanda, en la reducción de inventarios y en el aprovisionamiento justo a tiempo de insumos y materiales, ubican el punto crítico del 
proceso en la logística, en el control de los flujos, en las redes y, en última instancia, en la circulación. En términos de Coriat (1992), ahora lo comercial es lo que organiza y ordena los talleres, la lógica fordista es invertida, ya no se produce para vender, sólo se produce lo que está vendido.

En efecto, de acuerdo a este autor, las recientes innovaciones tecnológicas y organizativas dan lugar a un cambio fundamental en la búsqueda de la valorización. Ahora, "en vez de que la producción se haga en cadena desde el principio hasta el final del proceso, se hace desde el final hacia el principio del mismo. Esto significa que a partir de los pedidos hechos a la fábrica $y$, por lo tanto, de los productos efectivamente vendidos se programan las necesidades en unidades y en materiales" (Coriat, 1992: 50). Bajo esta perspectiva, pareciera reforzarse el papel de la circulación y ese imperativo de fluidez, propio del actual modelo de acumulación.

Algunos autores, con enfoques sistémicos sobre el análisis territorial y la geografía proclaman sin ningún recato la supremacía de la esfera de la circulación. En la fase de globalización, ... "como la circulación prevalece sobre la producción propiamente dicha, los flujos se han vuelto aún más importantes para la explicación de una determinada situación. La propia estructura geográfica se define por la circulación, ya que ésta, más numerosa, más densa, más extensa, ostenta el dominio de los cambios de valor en el espacio" (Santos, 2000: 227).

En definitiva, se requiere movilizar crecientes volúmenes de la producción fragmentada para poder integrarla, entonces hay que crear flujos, o si se quiere, fluidez y, para ello, se requieren redes cada vez más complejas y sofisticadas de transportes y comunicaciones para acelerar la movilidad de la mercancía y del capital. De esta manera “..., las redes constituyen los más eficaces transmisores del proceso de globalización al que asistimos" (Santos, 2000: 160).

En este sentido, la formación de la red global de puertos y transporte multimodal con sus nodos clave, los puertos concentradores (hubs), sólo fueron posibles por el cambio tecnológico y organizacional vinculado a la movilidad de la mercancía. En este sentido los puertos de la globalización son visualizados como nodos intermedios dentro de las redes de logística y transporte multimodal, que permiten articular cadenas de suministro globalizadas, desplegadas en localizaciones múltiples (Martner, 2020).

Precisamente, la eficacia de estas redes fundamentales para el proceso productivo globalizado son las que están siendo puestas a prueba, en primera instancia, por las tendencias neoproteccionistas y los amagos de guerra comercial entre países centrales de la economía-mundo y, en segunda instancia, por las fuertes disrupciones y sobresaltos ocasionados por la pandemia del COVID-19 sobre las extensas cadenas de suministros que se articulan a través del espacio global de redes y flujos.

\section{Tendencias comerciales previas al Covid-19 y sus repercusiones en el transporte marítimo y los puertos}

Sin duda, el modo de transporte más globalizado es el marítimo; más del $80 \%$ del comercio internacional se moviliza en buques mercantes y las cadenas de suministro más extensas (sean intercontinentales o intra-continentales) están vinculadas a las redes logísticas marítimo-portuarias. En este contexto, este modo de transporte es muy sensible a las variaciones del entorno económico internacional, a las políticas que estimulan o inhiben el comercio y a las dinámicas particulares de cada país. 
En las últimas décadas, un aspecto que ha influido en oscilaciones leves de los flujos tiene que ver con recurrentes desastres naturales como terremotos que derivan en tsunamis, ciclones y huracanes cada vez más intensos e inundaciones, muchos de los cuales, a decir de los expertos, están relacionados al cambio climático derivado del calentamiento global en curso. No obstante, estos eventos naturales reducen o paralizan la actividad marítimo-portuaria en puntos muy focalizados del planeta y por un corto periodo de tiempo (Martner et al, 2020).

Ahora bien, en el último tercio del decenio pasado emergieron con mayor fuerza tendencias proteccionistas e incluso aislacionistas en algunos países de gran peso en el concierto internacional, como Gran Bretaña y Estados Unidos, entre otros. El descontento de un proceso globalizador selectivo y que beneficio a sectores minoritarios dio lugar al surgimiento de diversas expresiones de rechazo, desde aquellas que buscan implementar políticas para atenuar la creciente desigualdad social y proporcionar oportunidades a los excluidos de la economía-mundo capitalista, hasta otras netamente regresivas que se amparan en banderas nacionalistas para tratar de reproducir supuestas grandezas pasadas, basadas en modelos anacrónicos, altamente jerárquicos y desiguales.

En este contexto, previo a la aparición del COVID-19 ya se observaba una tendencia decreciente de la economía y el comercio mundial, debido al progresivo avance de enfoques y políticas neoproteccionistas, marcadas por el condicionamiento y la modificación de acuerdos de libre comercio, por una escalada de aranceles punitivos (guerra comercial) y la consecuente inhibición de nuevas inversiones.

Así, durante 2019, las proyecciones del PIB mundial fueron revisadas constantemente a la baja. El reporte anual de la ONU de nombre Situación Económica Mundial (ONU,2020) señaló que la economía global registró, en 2019, su crecimiento más bajo de la década, cayendo a 2.3\%, como resultado de las prolongadas disputas comerciales y una desaceleración en la inversión global y doméstica. Por su parte, la Comisión Económica para América Latina y El Caribe (CEPAL), a principios del 2019, pronosticó un crecimiento de $1.7 \%$ en la región, pero en diciembre del mismo año estimó el crecimiento del PIB latinoamericano en sólo 0.1\% (CEPAL, 2019).

En 2018, todavía los puertos mexicanos registraban un robusto dinamismo de la carga comercial no petrolera, cuyo crecimiento fue de $5.2 \%$ con respecto al año anterior. En el caso de la carga contenedorizada el ritmo de crecimiento fue aún más alto, alcanzando un $9.7 \%$ anual.

No obstante, a partir de 2019, se manifestó un cambio de dirección de las tendencias previas, atribuible, en gran medida, al entorno turbulento de una guerra comercial que se manifestó a través de la imposición de aranceles principalmente de los Estados Unidos a China, pero también a otros países de Europa y de América Latina en productos específicos. Por ejemplo, en el caso mexicano se aplicaron aranceles sobre productos derivados del acero y el aluminio. La incertidumbre acerca de las nuevas reglas del comercio internacional a partir de estos afanes neoproteccionistas, así como de las modificaciones de los tratados de libre comercio hacia condiciones más restrictivas en tópicos relativos a las reglas de origen, entre otros, inhibieron el dinamismo económico y el movimiento de mercancías a nivel mundial.

Así, durante ese año, el comportamiento de la carga por puertos mexicanos comenzó a verse afectada al punto que la carga comercial no petrolera presentó una tasa de crecimiento negativa (-2\%) y la carga movida en contenedores creció sólo un $1.7 \%$ derivado de un comportamiento decreciente a partir del segundo semestre de ese año. En efecto, hasta agosto de 2019, los contenedores registraban todavía un aumento de $6.8 \%$ con respecto a igual periodo de 2018, sin embargo, a partir de ese mes se manifiesta una 
notable caída derivada de la intensificación de la política comercial proteccionista de elevación de aranceles, al punto de que, en el mes de diciembre de 2019, el movimiento de contenedores registró una caída de $12.5 \%$ con respecto al mismo mes del año previo (Figura 1).

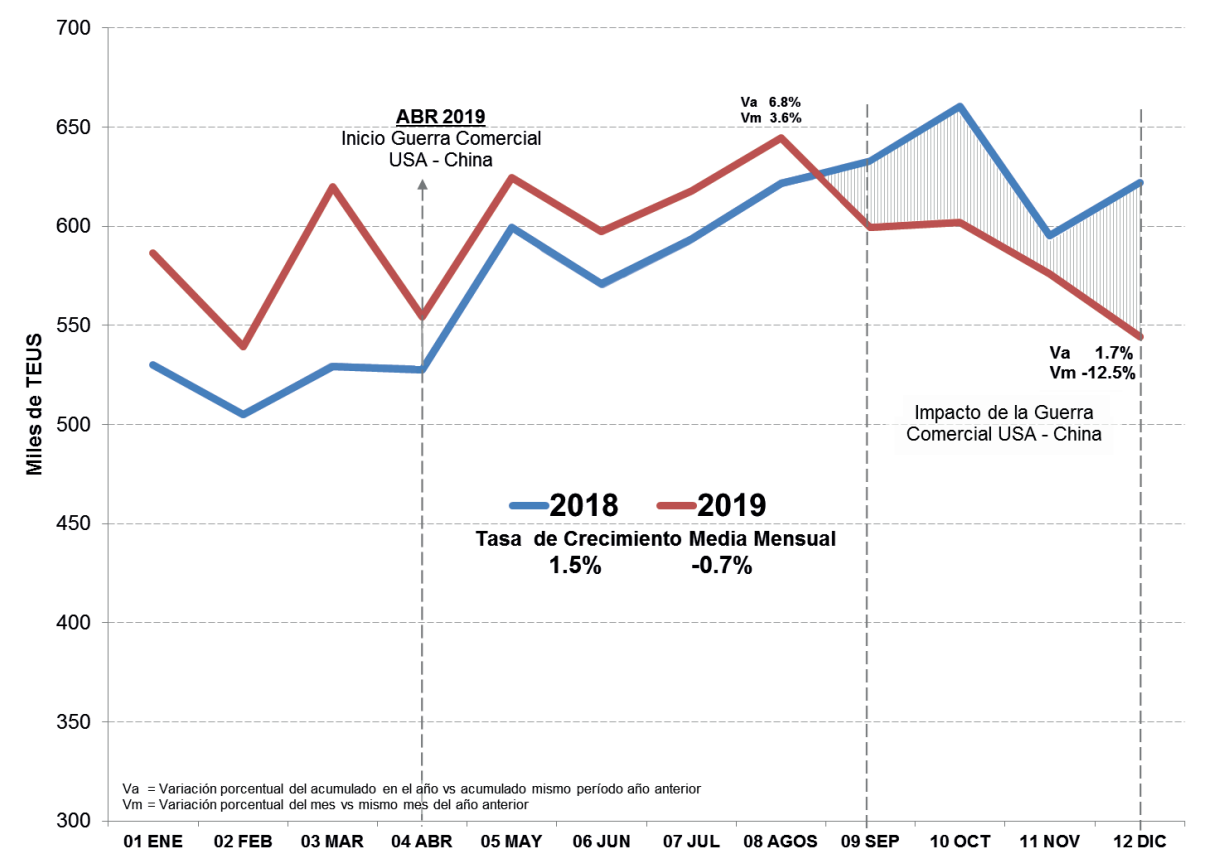

Figura 1. Evolución mensual de la carga contenerizada en los puertos mexicanos, 2018-2019 (TEUs). Fuente: Elaboración propia con base en datos de la Coordinación General de puertos y Marina Mercante-SCT.

\section{El Covid-19 profundiza la caída del comercio marítimo y "rompe" las cadenas de suministro globales}

Al entorno económico depresivo generado por la nueva ola de políticas proteccionistas y por la guerra comercial entre las grandes economías (especialmente, entre Estados Unidos y China), se sumó la progresiva expansión global del COVID-19, durante el primer trimestre de 2020.

A pesar de que, en enero de 2020, se registró un ligero repunte de los flujos, derivado de una disminución relativa de la tensión comercial internacional y de la conclusión de las negociaciones del nuevo tratado comercial entre Estados Unidos, México y Canadá, conocido como T-MEC, poco duró el gusto, puesto que, en febrero de ese año, comienzan a sentirse en los puertos mexicanos los primeros efectos del cierre de la economía China, debido al avance del COVID-19 en ese país asiático.

Los efectos económicos de esta pandemia se fueron escalonando progresivamente a medida que el COVID-19 avanzó sobre las distintas regiones y continentes. Primero se paralizaron las actividades productivas en Asia, mientras que las fábricas todavía seguían abiertas en América y el resto del mundo, pero no podían ser abastecidas por los insumos provenientes del continente asiático, con lo cual las cadenas de suministro desplegadas sobre el espacio global de redes y flujos fueron interrumpiéndose progresivamente. Posteriormente, Europa cerró sus actividades productivas y hacia finales de marzo de 2020 comenzó el confinamiento en el continente americano. 
Por el contrario, a partir de abril de 2020, los países asiáticos (en especial, China) comienzan a reabrir sus actividades productivas mientras el resto del mundo entraba en un riguroso confinamiento abocado a contener la pandemia, donde solo permanecieron abiertas las llamadas actividades esenciales, básicamente aquellas relacionadas con la salud, la alimentación y la energía.

Tales desajustes interrumpieron los ciclos normales de abastecimiento de bienes e insumos, alteraron los inventarios y evidenciaron la fragilidad las cadenas de suministro articuladas por las redes marítimo-portuarias en el espacio de flujos de la globalización. Desde marzo hasta junio de 2020 hubo un notable descenso del volumen de carga movilizado por el transporte marítimo internacional, acompañado por limitaciones y restricciones en la operación portuaria de muchos países. La Figura 2 muestra la caída de los flujos de carga contenedorizada en los puertos mexicanos durante el periodo señalado que corresponde a la fase de máximo confinamiento.

No obstante, un aspecto notable de esta crisis ha sido la capacidad demostrada por las líneas navieras regulares para adaptarse con gran rapidez a las fluctuaciones del mercado. Cuando muchos analistas presagiaban una debacle de este sector por la abrupta caída de los flujos comerciales y, con ello, un desplome de los fletes e ingresos, resultó sorprendente la notable flexibilidad y adaptación de estas empresas de transporte para ajustar la oferta a la demanda.

Esta reacción evitó la caída abrupta de las tarifas e incluso, en muchas de las rutas principales, se observó un notable aumento de los fletes cobrados. Entonces ¿Cómo entender esta tendencia? Al menos hay dos elementos explicativos que resultan relevantes. Por un lado, el largo proceso de consolidación de las líneas navieras regulares de contenedores y la formación de alianzas estratégicas entre ellas les ha permitido tener una visión más amplia del mercado y, por otro lado, la digitalización de las operaciones ha sido un factor crucial para obtener enormes cantidades de información oportuna que les permitió ajustarse con mayor agilidad a las fluctuaciones de la demanda.

En otras palabras, ahora hay menos navieras que hace una década y éstas forman parte de grandes consorcios y/o alianzas cuasi oligopólicas, lo cual les permite tomar decisiones de manera mucho más fácil. Adicionalmente, tienen más instrumentos tecnológicos para predecir el comportamiento de la demanda, de tal modo que pudieron saber anticipadamente cuántos embarques exactamente estaban cancelando sus cargadores y, a partir de ahí, eliminaron viajes en rutas programadas (lo que se conoce como blank saling), evitando una sobreoferta de capacidad que derrumbaría los fletes.

No obstante, como en todo pronóstico, hubo elementos no controlados y en varias rutas la demanda, de por si errática en tiempos de pandemia, fue mayor a lo esperado por las navieras, es decir, a los viajes cancelados (blank sailing), generando inquietantes incrementos de tarifas para los cargadores. 


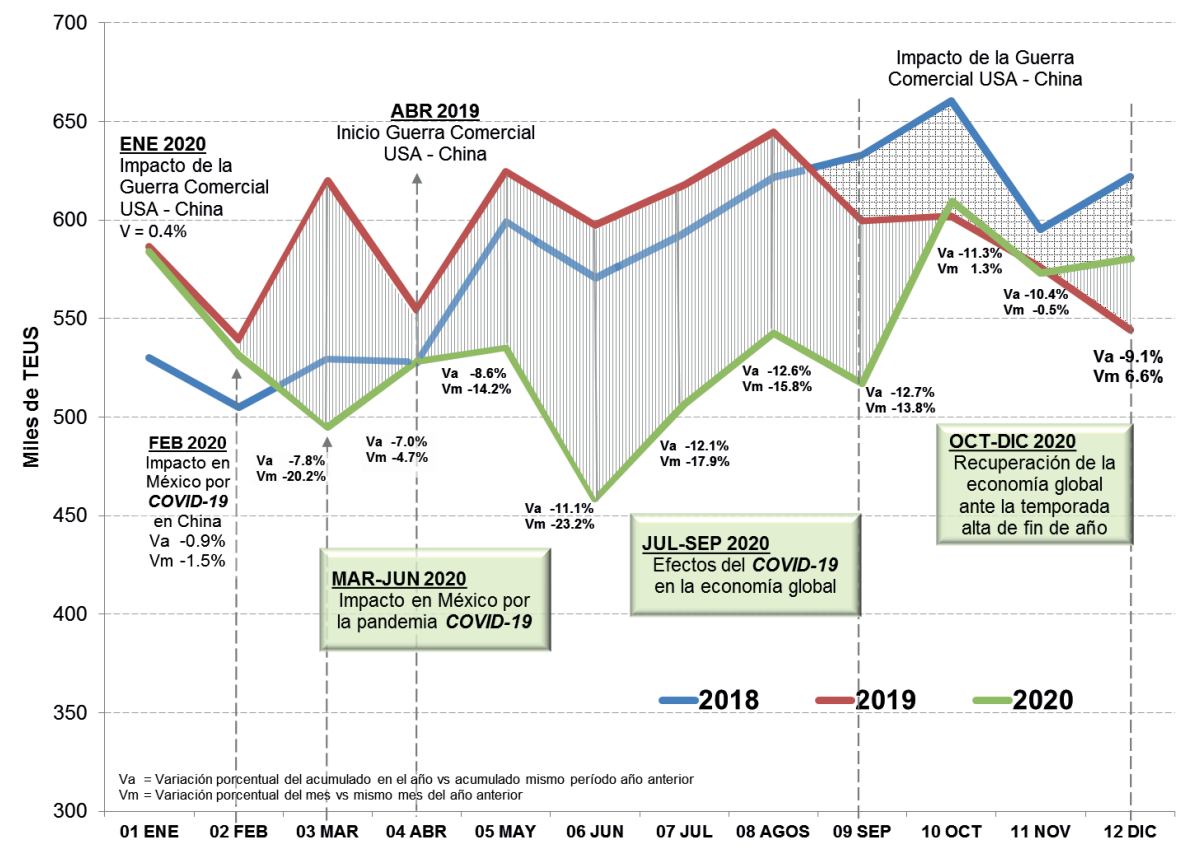

Figura 2. Evolución mensual de la carga contenerizada en los puertos mexicanos, 2018-2020 (TEUs). Fuente: Elaboración propia con base en datos de la Coordinación General de puertos y Marina Mercante-SCT.

\section{Recuperación de los flujos de carga en la fase de reapertura progresiva de las actividades económicas}

La reapertura progresiva de las actividades económicas estimuló la activación de la demanda de embarques. A partir de junio de 2020, en los puertos mexicanos se observó una recuperación significativa de los flujos de carga e inclusive durante el último trimestre del año las cifras del movimiento de contenedores en las costas nacionales superaron a las de 2019 (Figura 2). Este impulso se ha mantenido durante los primeros meses del 2021, superando el nivel de movimiento de carga previo a la pandemia.

Este proceso de recuperación de flujos se dio todavía con más fuerza en las economías desarrolladas, donde se desató un boom de demanda de bienes manufacturados sin precedentes, el cual está ocasionando nuevas disrupciones en las cadenas de suministro marítimo-portuarias. A pesar de que la pandemia del COVID-19 no ha sido superada y muchas actividades mantienen restricciones, debido al incremento de contagios a nivel mundial, paradójicamente la demanda de bienes de consumo duradero se disparó alcanzando niveles record. ¿Cómo explicar esta paradoja del comercio internacional y de las cadenas de suministro globalizadas movilizadas por el trasporte marítimo e intermodal de contenedores?

\section{Modificación de los patrones de consumo, desequilibrios y restructuración de los flujos marítimos e intermodales}

Entre los cambios generados por la pandemia en la vida diaria de la población, destaca la llamada al encierro por parte de las autoridades sanitarias, el cierre de negocios enfocados a los servicios, tales como restaurantes, bares, gimnasios, centros culturales y de esparcimiento, la necesidad de las empresas e instituciones públicas y privadas de cumplir con sus objetivos sin tener a los trabajadores en contacto directo, orillándolas 
al home office y la baja necesidad de trasladarse cotidianamente cambiaron los hábitos de consumo considerados normales hasta ese momento (Goodman et al, 2021).

A su vez, de acuerdo a Miller (2020) las redes digitales y tiendas en línea abrieron paso a una ola de pedidos de diversos artículos que se ajustaban a la nueva normalidad del consumidor, ahora debía adecuar los espacios de su casa para trabajar, ejercitarse o simplemente remodelar o amueblar con el dinero con que disponía, mismo que no podía gastar en salidas o viajes. Adicionalmente, los estímulos económicos implementados en algunos países con el fin de no detener el flujo activo de capital, aumentaron las posibilidades de sus habitantes de mantener o incrementar la demanda de bienes.

En este sentido, se advierte que la pandemia está teniendo un impacto significativo sobre la modificación de los patrones de consumo de la población. Ante las restricciones para el consumo de actividades relacionadas con los servicios (viajes, vacaciones, actividades recreativas, deportivas, espectáculos, cultura, asistencia a gimnasios, restaurantes, cines, etc.), el consumo se ha concentrado en bienes relacionados con el confinamiento, el trabajo en casa, aparatos de acondicionamiento físico, muebles y equipos electrónicos para la conectividad virtual, así como todos los bienes relacionados con el cuidado de la salud y la prevención del contagio.

De esta forma, la pronosticada baja actividad en el transporte marítimo e intermodal de bienes fue desmentida rápidamente por la nueva realidad del consumo durante el último cuatrimestre del año 2020 y lo que va del 2021. Efectivamente, tal cambio en los patrones de consumo se reflejó en el incremento acelerado de bienes producidos principalmente en China y el Lejano Oriente con destino a los principales mercados de Europa y América. La necesidad de reponer bajos inventarios en los principales mercados de consumo y de atender los crecientes requerimientos de productos propios del nuevo patrón de consumo, evidenció la insuficiencia del transporte marítimo internacional para ajustarse a un crecimiento exponencial de la demanda.

La demanda de bienes superó la capacidad que tienen las redes de suministros establecidas. Pese a la guerra comercial, la pandemia y el cierre de actividades, las empresas navieras y de transporte multimodal percibieron grandes ganancias provocadas por el volumen de proveedores de las cadenas de suministro que necesitaban hacer llegar sus productos a los consumidores, muchos de ellos situados en los países desarrollados, pero también en segmentos de los llamados países de renta media o emergentes, lo que, a su vez, sobrepasó el aforo de los puertos.

\section{Los problemas de articulación modal y espacial de las cadenas de suministro conectadas por los puertos}

El sistema de transporte en contenedores, aunque versátil y asequible, no está en condiciones de soportar un evento inesperado de hiper crecimiento de los flujos de bienes en poco tiempo por un sorpresivo y acelerado cambio de los patrones de consumo derivado de la pandemia. Actualmente, los principales puertos del mundo están sobrecargados, tienen a los buques flotando en la cercanía con todos los bienes esperando ser descargados (Goodman et al, 2021).

Los contenedores escasearon, las rutas y la disponibilidad de espacio en los buques fueron insuficientes, las demoras para la atención de las embarcaciones en los puertos de destino crecieron enormemente, derivado del incremento de los arribos y de las dificultades de las terminales que no solo están lidiando con el abrupto crecimiento del volumen de trabajo, sino con la disponibilidad de la plantilla de trabajadores que sufre los embates de la pandemia constantemente. De igual forma, en la parte terrestre de la cadena logística-portuaria, hay afectaciones notables por falta de camiones 
y carros de ferrocarril para atender la burbuja de demanda de los últimos meses, con lo cual las dificultades para las entregas justo a tiempo de los insumos y productos transportados son enormes.

Las alternativas tomadas hasta el momento para compensar el problema de desabasto en puertos han sido varias, por una parte, los productores comenzaron a utilizar otros medios de transporte, como el aéreo, lo que aumentó sus costos de producción, pero permitió mejorar parcialmente los tiempos de entrega.

$\mathrm{Al}$ respecto, un caso icónico es el de la empresa transnacional Peloton, especializada en la producción de bicicletas fijas y sus partes, cuya cadena de suministro desplegada en el espacio global se desarticuló por un crecimiento de la demanda de $128 \%$ durante el último cuatrimestre de 2020, dado que no encontraba contenedores ni barcos para hacer llegar a tiempo el producto de Asia a América. Ante la creciente queja de los consumidores por retrasos que se extendieron a meses en lugar de semanas, dicha empresa tuvo que buscar variantes en las redes de transportes para mitigar las demoras, incrementando los costos de traslado tanto por la elevación de los fletes marítimos e intermodal, como por el envío vía aérea de los pedidos de mayor urgencia (Straight, 2021).

Cabe señalar que, de acuerdo a las principales revistas especializadas de transporte marítimo e intermodal internacional, este tipo de relatos se está repitiendo para el caso de múltiples ramas de la industria manufacturera de bienes de consumo duradero, como la automotriz, la electrónica, la de calzado y vestido, de muebles, de alimentos, de bebidas y de diversos artículos para el hogar y la salud.

Este círculo vicioso expresado en la falta de contenedores para embarcar los productos, demoras y congestión en los puertos de origen y destino, ausencia de personal e insuficiente disponibilidad de transporte terrestre se expresa en al menos dos problemas sustantivos para las cadenas de suministro marítimo-portuarias que se despliegan sobre el espacio reticular de la red global. Por una parte, los precios de los servicios marítimos e intermodales se han incrementado de manera sustantiva, provocando alzas que, en ocasiones, cuadriplican las tarifas por cada contendor movilizado, por otra parte, se advierte una fuerte caída de la confiabilidad de los tiempos de arribo de las embarcaciones a los puertos de destino.

\section{Altos costos y baja confiabilidad de las cadenas de transporte marítimo e intermodal globalizadas: Otra consecuencia de la pandemia}

Las cadenas de suministro extensas articuladas en el espacio continental o global por el transporte marítimo e intermodal enfrentan en la actualidad elevados costos de envío y poca certeza en los tiempos de entrega de los embarques, rompiendo con conceptos básicos de la logística de distribución de bienes intermedios y de consumo final y de la rentabilidad de la producción fragmentada a escala internacional, donde el manejo de inventarios justo a tiempo o justo en secuencia (Cedillo et al, 2019) forma parte de la eficiencia y la competitividad de tales cadenas productivas extendidas en el espacio de redes y flujos de la globalización.

El alza en la demanda de transporte de mercancías, a partir del último tercio del año 2020, propició la elevación sustantiva de las tarifas por parte de las navieras internacionales. Las rutas comerciales más solicitadas pagan precios inflados para cubrir sus necesidades de traslado y, a su vez, congestionan con buques cargados las costas de estas regiones, mientras que las menos favorecidas, entre las que se incluyen zonas de América Latina (también con tarifas elevadas) no cuentan con suficientes naves para garantizar un correcto envío de sus materias primas y bienes (Villahermosa, 2021). 
La Figura 3 muestra el notorio incremento de tarifas por contenedor en las dos principales rutas transpacíficas, esto es, desde Asia a la Costa Oeste (Pacifico) de Estados Unidos y desde el continente asiático a la Costa Este de Estados Unidos (Atlántico) vía el Canal de Panamá. De mayo de 2020 a abril de 2021, los fletes se han triplicado, pasando de alrededor de 1 mil 600 dólares por contenedor a más de 5 mil dólares en el Pacífico.

Todavía resultan más sorprendentes los aumentos de tarifas en países no centrales dentro de la economía-mundo capitalista. Tal como lo ha revelado el informe titulado Policy Brief de la UNCTAD (2021), el efecto de encarecimiento de los fletes ha sido más grande en países latinoamericanos y en otras regiones periféricas cuya demanda de bienes manufacturados es notoriamente menor que la de los países centrales.

Según dicho informe, parte de la explicación radica en el hecho de que las rutas desde China y el Lejano Oriente a países de regiones como Sudamérica, Centroamérica y África suelen ser más largas.

Se requieren más barcos para el servicio semanal en estas rutas, lo que significa que muchos contenedores también están "atascados" en estas rutas. Cuando los contenedores vacíos son escasos, un importador en Brasil o Nigeria debe pagar no solo por el transporte del contenedor de importación completo, sino también por el costo de mantenimiento del inventario del contenedor vacío. Otro factor puede ser la falta de carga de regreso. Las naciones de América del Sur y África occidental importan más productos manufacturados de los que exportan, y es costoso para los transportistas devolver cajas vacías a China en rutas largas (UNCTAD, 2021:3).

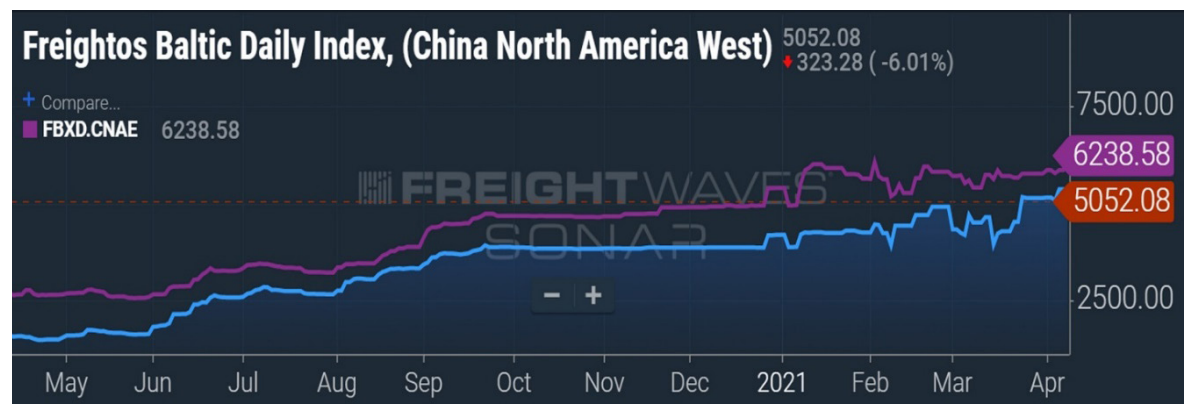

Figura 3. Tarifa por Contenedor de 40 pies desde China a las Costa Oeste (Pacífico) y Este (Atlántico) de Estados Unidos (2020-2021). Fuente: FreightWaves 2021.

En el caso mexicano, el incremento de tarifas es notablemente superior al de los Estados Unidos. De acuerdo a datos de EAX Eternity México (2021), a mediados del año 2020, la tarifas por contenedor de 40 pies desde Asia a los puertos mexicanos del Pacífico (Manzanillo y Lázaro Cárdenas) eran inferiores a los 1 mil 500 dólares. En diciembre de 2020, alcanzó los 6 mil dólares, es decir se cuadriplicó. Para febrero de 2021, subió a 7 mil 600 dólares por contenedor (figura 4), es decir, quintuplicó en menos de un año, debido a las disrupciones de las cadenas de suministro derivadas de la pandemia que se han señalado a lo largo de este artículo.

En todo caso, no deja de ser paradójico el hecho que quienes menos tienen que ver con el boom de la demanda actual y sus disrupciones (demoras excesivas, la falta de equipo, la saturación de puertos y retrasos considerables en el abastecimiento de las cadenas de suministro fragmentadas) sean los que mayores sobrecostos enfrenten en la actualidad, afectando de manera más significativa la competitividad de los eslabones de las cadenas de suministro que se encuentran en los países emergentes o no centrales de la economía-mundo. 


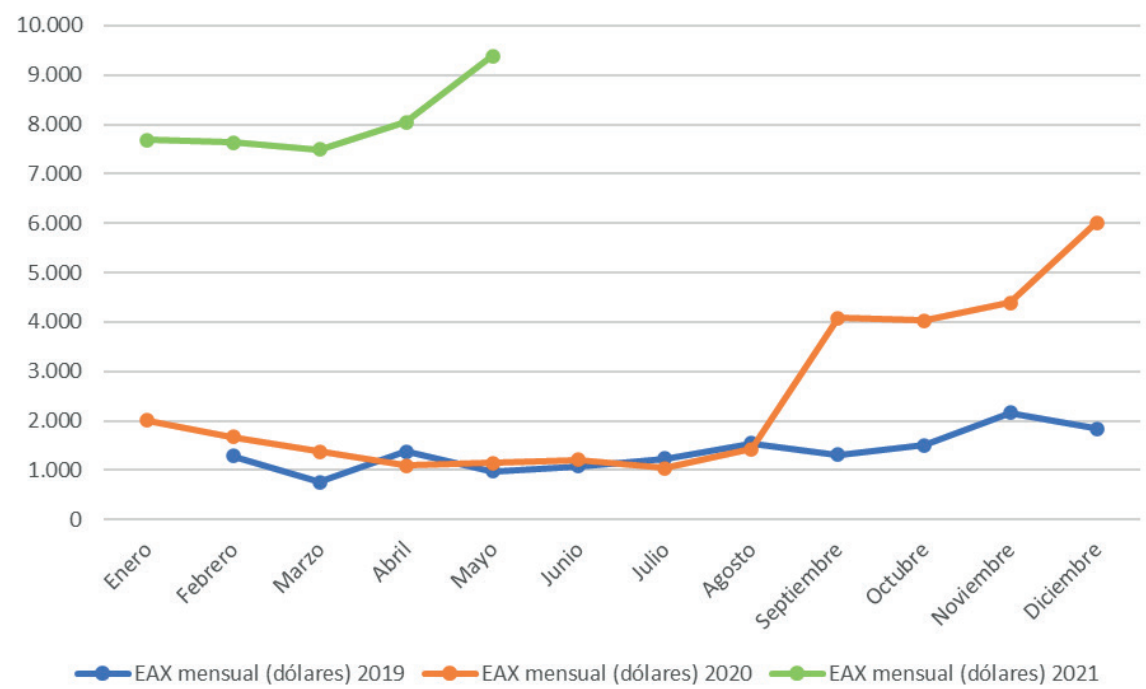

Figura 4. Tarifas Marítimas por contenedor de 40 pies entre Asia y los puertos del Pacífico Mexicano (Manzanillo y Lázaro Cárdenas). Fuente: Elaboración propia con base en datos de Eternity México.

El otro aspecto que está teniendo consecuencias sobre la producción fragmentada desplegada en el espacio de redes y flujos de la globalización, es el de la confiabilidad del arribo de los insumos y productos a su destino. En efecto, se advierte una fuerte caída de este indicador relativo a los tiempos de arribo de las embarcaciones. Durante 2018 y 2019 las llegadas a tiempo de los buques de contenedores se ubicaban en torno a la franja del 75 al $80 \%$ a nivel global, no obstante, a partir de agosto de 2020 se observó un deterioro sostenido, al punto que, en diciembre del año pasado, la confiabilidad cayó a un $45 \%$ de llegadas a tiempo. El último dato disponible, correspondiente a abril de 2021, refleja un deterioro todavía mayor de la confiabilidad de las llegadas a tiempo de los barcos portacontenedores, al alcanzar sólo el 39.2\% (Figura 5), lo que demuestra que los desajustes en las cadenas de suministro marítimo-portuarias están lejos de amortiguarse y los nuevos patrones de consumo mantienen cotas altas de demanda de bienes de consumo manufacturados.

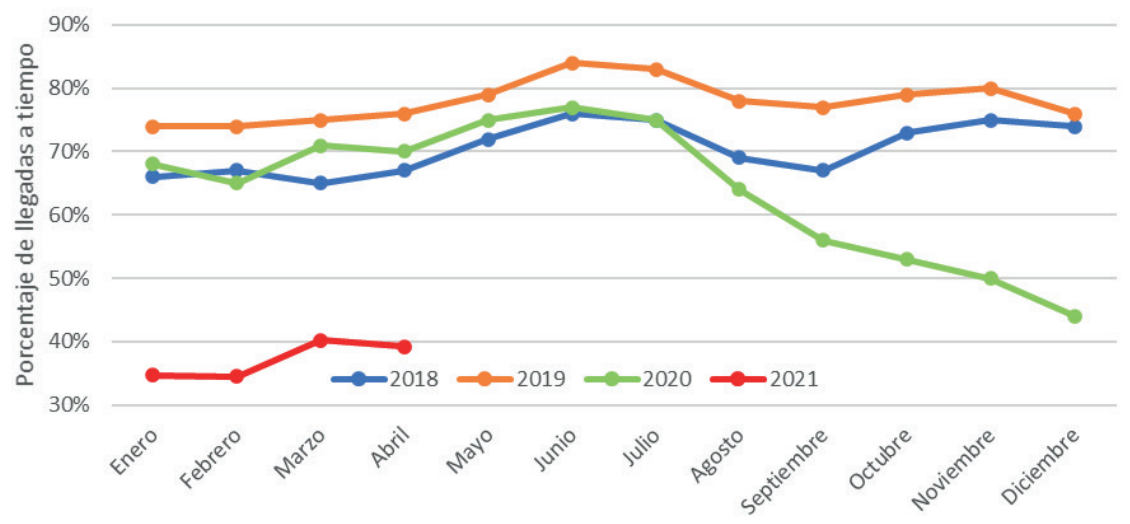

Figura 5. Nivel de confiabilidad del arribo de buques portacontenedores a nivel global, 2018-2021. Fuente: Elaboración propia con base en datos de Sea Intelligence Consulting.

De acuerdo con los datos de Sea Iintelligence (Think Goblal Logistic, 2021), la confiabilidad del transporte marítimo de contenedores entre Asia y Estados Unidos estuvo 
muy por debajo del promedio mundial. Para octubre-noviembre, las llegadas a tiempo se redujeron al 28,6\% en el comercio Asia-Costa Oeste y al 26,4\% en el comercio Asia-Costa Este. Esto ha quedado de manifiesto en las notas de revistas especializadas y en imágenes donde se observan veintenas de buques portacontenedores fondeados durante muchos días en la bahía de Los Ángeles, esperando poder entrar a descargar en los puertos del sur de California.

En este contexto, donde han proliferado las disrupciones y se han manifestado vulnerabilidades constantes de las cadenas de suministro globales, que se expresan en interrupciones de las líneas de producción, insuficientes inventarios, demoras excesivas, sobrecostos incrementales y bajo nivel de confiabilidad para la entrega de embarques en el destino final, se cuestiona crecientemente la eficacia de la globalización con sus extensas cadenas de suministro que requieren ser integradas espacialmente salvando enormes distancias entre los puntos de origen y destino de los bienes movilizados. Dada las vulnerabilidades que puso de manifiesto la pandemia del COVID-19 ha resurgido el debate sobre la relocalización y regionalización de las cadenas de suministro como una forma de solucionar los desafíos presentes.

\section{Regionalización versus globalización ¿un dilema real?}

A medida que los efectos de la pandemia del COVID-19 fueron afectando la confiabilidad de las cadenas de suministro articuladas por el transporte marítimo e intermodal, resurgieron los planteamientos sobre una posible desglobalización de las actividades económicas y el fortalecimiento de una regionalización basada en una mayor cercanía física entre proveedores de insumos, fabricantes y zonas de consumo. El término de nearshoring volvió a ser recurrente en notas y artículos sobre logística (Altman et al, 2021), puesto que al acercar los proveedores a los puntos de producción y consumo se puede reducir notablemente la vulnerabilidad de las cadenas de suministro frente a eventos disruptivos como las pandemias, los desastres naturales y las guerras comerciales.

Como categoría de análisis, el término de nearshoring surge en las facultades de negocios internacionales de diversas universidades estadounidenses, en contraposición al término comercial offshoring, este último entendido como la deslocalización de fases del proceso productivo en ubicaciones lejanas o transcontinentales, pero que generan enormes ventajas competitivas por reducción de costos de producción principalmente, cuestión que ha constituido la base de la globalización. A diferencia de ello, el nearshoring se refiere a la transferencia de fases de los procesos productivos y/o comerciales a un país cercano (Miller, 2020b), donde las cadenas de suministro se beneficiarían de una o más de las siguientes dimensiones de proximidad: vínculos geográficos, temporales (zona horaria), culturales, lingüísticos, económicos, políticos o históricos.

El deseo de reconfigurar las regiones que componen las cadenas de suministro, una opción explorada previamente, pero dejada de lado por los incrementos de costos que implicaba dicha solución, retoma fuerza en este momento donde las disrupciones de las cadenas productivas ocasionadas por la pandemia están generando enormes sobrecostos y perdidas. En este contexto, se plantean aspectos como la necesidad de reducir las distancias que deben recorrer los bienes, o la manera en que se tendrían que articular los modos de transporte marítimo y terrestre para construir redes intermodales más resilientes ante eventos disruptivos como las pandemias y los desastres naturales.

Adicionalmente, los temas relacionados con la agenda internacional para controlar la emisión de gases efecto invernadero y el calentamiento global constituirían, eventualmente, otro incentivo para el nearshoring, en la medida que se reduciría la distancia de 
los viajes al reforzar los aprovisionamientos de proximidad. No obstante, cabe señalar que este factor ambiental también puede ser atenuado mediante el avance la agenda de descarbonización del transporte marítimo que, en la actualidad, impulsa fuertemente la Organización Marítima Internacional (OMI).

Lo cierto es que las fracturas ocasionadas por el COVID-19 a la estructura y el núcleo de las cadenas productivas sustentadas sobre la base de un espacio globalizado de redes y flujos de tipo offshoring, instala en el debate la posibilidad de una nueva reconfiguración territorial donde la regionalización de los sistemas productivos cobra relevancia.

Y es que se ha visto que durante el confinamiento escalonado de actividades productivas en diversas regiones del mundo muchos fabricantes de automóviles, equipos electrónicos, maquinaria, vestido y calzado, entre otros, dependen de las entregas justo a tiempo para sus líneas de montaje. Esas líneas de montaje se detuvieron por completo mucho más rápido de lo que nadie esperaba, lo cual evidenció vulnerabilidades importantes que pusieron en jaque el actual modelo de producción espacialmente fragmentado, basado en cadenas de suministro geográficamente extendidas.

$\mathrm{Al}$ respecto, Miller (2020b) señala que, con la guerra comercial entre Estados Unidos y China, ya se había evidenciado una tendencia hacia una relativa descentralización de cadenas de suministro alojadas en China, que comenzaron a llevar eslabones del proceso de fabricación a países vecinos como Camboya, Vietnam, Filipinas y Malasia para eliminar el riesgo de verse afectados por altos aranceles. Sin embargo, esto no ha implicado acortar las distancias con respecto a los principales mercados de destino de esos productos, los cuales se ubican en Norteamérica y Europa.

Para el propio Miller (2020b) en lo inmediato los importadores compensarán los riesgos de la cadena de suministro asumiendo más costos de inventario y enviando más volumen a los almacenes y centros de distribución en los países de destino. Pero cuando se trata de cambiar los países donde se producen los bienes manufacturados que se movilizan en contenedores, es difícil que ello ocurra en medio de una pandemia.

En cuanto a las perspectivas de la relocalización de la cadena de suministro global como consecuencia de la pandemia y su impacto en el transporte de contenedores, algunos autores señalan que ciertamente habrá un cierto nivel de regionalización y muchas cosas cambiarán, pero de ninguna manera se espera un cambio rápido o total de paradigma, porque no todos los países son capaces de producir de manera eficiente y competitiva la enorme cantidad de partes, insumos y bienes intermedios que integran cada uno de los productos finales (Altman et al, 2021).

En efecto, la realidad es más compleja, las capacidades competitivas de los países no se desarrollan de la noche a la mañana. Producir a costos competitivos, con el suficiente nivel técnico y la disponibilidad de personal calificado supone un desarrollo de largo plazo, con políticas públicas encaminadas a ese objetivo que normalmente cristalizan en décadas.

Por tal razón, hablar de desglobalización en el corto y mediano plazo resulta al menos exagerado. El reciente boom de importaciones chinas en Estados Unidos demuestra que, a pesar de un entorno previo de guerra comercial, ambas economías se encuentran sumamente integradas.

La disociación entre Estados Unidos y China ha avanzado algo desde el inicio de la guerra comercial en 2018, pero estas economías siguen estando muy entrelazadas. La participación de China en el comercio de EE. UU. se disparó durante la pandemia, y las multinacionales estadounidenses como Walmart, Tesla, Disney y Starbucks 
continúan invirtiendo allí. Además, la distancia promedio a través de la cual los países comercian ha tenido una modesta tendencia ascendente desde 2016. Esto arroja dudas sobre el argumento de que estamos viendo un gran cambio de la globalización a la regionalización (Altman et al, 2021:3).

En este sentido, el nearshoring o regionalización de las cadenas productivas tiene límites objetivos que dificultarán en el corto y mediano plazo un traslado masivo de plantas y actividades de un continente a otro, de una región a otra. Es decir, la regionalización probablemente avanzará en algunas fases de la cadena de suministro y en algunos bienes específicos, pero a un ritmo lento. Entonces cabe preguntarse ¿qué opciones existen para disminuir la vulnerabilidad de las cadenas de suministros extensas? Entre estas se plantea la diversificación de las fuentes de aprovisionamiento para no ser tan dependiente de unos pocos proveedores ubicados en una sola región.

Este nuevo tipo de flexibilización, tanto técnica como espacial, podría proporcionar mayor robustez a las cadenas de suministro articuladas por el transporte marítimo e intermodal y daría la posibilidad para que algunos países Latinoamericanos pudiesen captar parte de tal diversificación multiregional de proveedores que busca tener más opciones accesibles de insumos y bienes intermedios, acortar relativamente las distancias y hacer menos vulnerable el proceso de producción fragmentado a escala global. Sin duda, México, con el nuevo tratado comercial de Norteamérica (el T-MEC) aparece geográficamente como una localización con posibilidades de captar eslabones de ese proceso de diversificación de los lugares de proveeduría de insumos para las cadenas productivas fragmentadas a escala internacional.

El segundo aspecto relevante se relaciona a la necesidad de gestionar los inventarios con un mayor número de centros de distribución cercanos a los mercados finales. Esto parece estar siendo considerado seriamente, ya que las demoras e incumplimientos de entrega están generando costos muy superiores que los que pudiese tener cualquier inventario de seguridad.

En tercer lugar, se plantea la urgencia de desarrollar planes de contingencia flexibles y de avanzar con mayor rapidez en la visualización y trazabilidad en tiempo real de la cadena marítima e intermodal completa, desde la puerta de la planta en el país de origen hasta la puerta de la planta en el país de destino, a través de la digitalización de procesos en plataformas distribuidas, tipo Blockchain o similar, que permitan realizar análisis de datos masivos (Big Data) en diferentes segmentos de la cadena logística, más allá del tramo marítimo, pues claramente, el boom de demanda reciente en Estados Unidos y otros países, se relacionó con un problema de bajos inventarios que no habían podido reponerse durante meses y con un nuevo patrón de consumo derivado de la pandemia.

Estos cambios deben estar encaminados a mejorar las condiciones de toda la cadena de suministro, si bien una intención inicial sería buscar la relocalización de actividades que puedan realizarse de manera competitiva en el país o la región de pertenencia y, de esta manera, reducir distancias y articular de manera eficaz el trayecto de estas nuevas rutas, también se debe considerar mejorar las condiciones laborales de los trabajadores de este sector, incluidas aquellas que garanticen su salud en condiciones de riesgo como las actualmente existentes.

\section{Reflexiones finales}

La pandemia del COVID-19 ha golpeado la estabilidad de las cadenas de suministro marítimo-portuarias e intermodales conformadas dentro de la lógica de un espacio 
reticular globalizado que, tecnológicamente, permite fragmentar el proceso productivo en eslabones localizados en diversas regiones y territorios del mundo, dependiendo de la rentabilidad susceptible de obtener en cada uno de ellos.

El cierre escalonado de actividades económicas en los distintos continentes, así como el desconfinamiento diferenciado temporal y regionalmente, junto con los cambios en los patrones de consumo de la población, produjeron, en la primera mitad del 2020, una caída pronunciada de los flujos de carga manejada por los puertos mexicanos (y a nivel mundial) que se revirtió vertiginosamente en la segunda parte de ese año y, en especial, en el último cuatrimestre. El sistema de transporte marítimo e intermodal todavía no ha podido ajustarse a tales desequilibrios. A mediados de 2021, la demanda todavía está lejos de equilibrarse con la oferta y los patrones de consumo recientes no parecen modificarse sustancialmente a pesar del avance en el proceso de desconfinamiento por vacunación sobre todo en los países desarrollados. Probablemente, en el mediano plazo, con la incorporación de más buques y la relativa atenuación de la demanda bienes transables a favor de un mayor gasto en servicios de esparcimiento y recreación, retorne cierto equilibrio en las cadenas de suministro marítimo-portuarias, aunque todo apunta a que esto no sucederá antes del 2022.

Tal como se señaló en el análisis previo, la caída inicial de los flujos y el posterior boom de la demanda de bienes manufacturados, relacionada sobretodo con las economías desarrolladas, pero con afectaciones globales, está provocando insuficiencia de buques y falta de contenedores para embarcar los productos, incremento de las demoras y congestión en los puertos de origen y destino, ausencia de personal afectado por el Covid-19 e insuficiente disponibilidad de transporte terrestre. Todo ello se expresa todavía en al menos dos problemas sustantivos para las cadenas de suministro marítimo-portuarias que se despliegan sobre el espacio reticular de la red global. Por una parte, los precios de los servicios marítimos e intermodales se han incrementado de manera sustantiva, provocando alzas que, en ocasiones, cuadriplican o quintuplican las tarifas por cada contenedor movilizado y, por otra parte, se advierte una dramática caída de la confiabilidad de los tiempos de arribo de las embarcaciones a los puertos de destino, con lo cual los insumos, bienes intermedios y productos no están llegando a tiempo a las líneas de producción ni a los puntos de venta final. Esto genera falta de disponibilidad de materiales para mantener la continuidad de los procesos productivos, enormes demoras y sobrecostos en la cadena logística de transporte y distribución y una perdida sustantiva de competitividad basada en el modelo de fragmentación de los eslabones de las cadenas de suministro sobre el espacio global.

En este contexto, se ha abierto el debate sobre la posibilidad de una suerte de desglobalización y de una reconfiguración espacial donde la regionalización de los sistemas productivos y las cadenas de suministro mundializadas cobra relevancia. $\mathrm{Al}$ respecto, se plantea que habrá un cierto nivel de regionalización, pero de ninguna manera se espera un cambio sustantivo en el corto ni en el mediano plazo.

Efectivamente, no todos los países son capaces de producir de manera eficiente la enorme cantidad de partes, insumos y bienes intermedios que integran cada uno de los productos finales. Producir a costos competitivos, con el suficiente nivel técnico y la disponibilidad de personal calificado supone un desarrollo de largo plazo, con políticas públicas encaminadas a ese objetivo que normalmente cristalizan en décadas.

Así, la regionalización de las cadenas productivas tiene límites objetivos que dificultarán en el corto y mediano plazo un traslado masivo de plantas y actividades de un continente a otro, de una región a otra. 
Por eso, frente a las disrupciones ocasionadas por el neoproteccionismo (guerra comercial) y el Covid-19, más que una nueva regionalización, se plantea la diversificación de las fuentes de aprovisionamiento para no ser tan dependiente de unos pocos proveedores ubicados en una sola región.

Este nuevo tipo de flexibilización, tanto técnica como espacial, podría proporcionar mayor robustez a las cadenas de suministro articuladas por el transporte marítimo e intermodal y daría la posibilidad para que algunos países Latinoamericanos pudiesen captar parte de tal diversificación multi-regional de proveedores que busca tener más opciones accesibles de insumos y bienes intermedios y hacer menos vulnerable el proceso de producción fragmentado a escala global.

Sin duda, México, con el nuevo tratado comercial de Norteamérica (el T-MEC) aparece geográficamente como una localización con posibilidades de captar eslabones de ese proceso de diversificación de los lugares de proveeduría de insumos para las cadenas productivas fragmentadas a escala internacional. En trabajos posteriores, habrá que seguir de cerca la evolución de estas tendencias emergentes que apenas se bosquejan para identificar los impactos sobre los puertos, la logística, las redes de transporte intermodal y el territorio.

Por lo pronto, parece inevitable la necesidad de transitar hacia esquemas más flexibles y resilientes, entre los que destacan la aplicación y/o creación de planes de contingencia, la priorización de los servicios esenciales, la reorganización de las operaciones y condiciones de trabajo atendiendo protocolos sanitarios y el desarrollo de estrategias de digitalización e intercambio de información en plataformas distribuidas.

Sin duda, la digitalización de las interacciones y el intercambio de información es otra tendencia emergente relevante a observar, dado que ha sido fundamental para la continuidad de las operaciones de transporte marítimo durante la pandemia, aunque insuficiente para resolver las disrupciones por la sobredemanda de contenedores de los últimos meses, así como por las demoras y congestión portuaria y terrestre de las cadenas intermodales.

Los cargadores y los puertos han trabajado para abordar las operaciones en tierra, pero la capacidad de adaptación no siempre ha sido eficaz. Resulta cada vez más evidente que para responder a los desafíos del COVID-19 se necesita desarrollar una visión sistémica de toda la cadena logística marítimo-portuaria que vaya más allá de los tramos navegables y de los puertos.

En efecto, la capacidad de coordinarse con las autoridades de los distintos niveles de gobierno y de comunicarse con otros actores de la parte terrestre a lo largo de toda la cadena es un desafío enorme, pero fundamental para construir redes resilientes en un entorno sumamente volátil y cambiante derivado de las circunstancias actuales.

Sin duda, una de las tendencias más relevantes en el futuro próximo será generar trazabilidad y visibilidad en tiempo real para cada eslabón de la cadena de suministro, con el fin de que los actores clave puedan tomar decisiones rápidas sobre posibles obstáculos, demoras, sobrecostos y perdidas de valor para el conjunto de la economía local, regional y nacional. 


\section{Q Bibliografía}

"Castells, M. (2021/06/23). Globalisation, networking, urbanisation: reflections on the spatial dynamics of the information age. Urban studies journal limited (2737-2745). Recuperado de https://doi.org/10.1177/0042098010377365 (16/11/2010).

»Castells, M. (2006). La Sociedad Red. 2da edición. Madrid: Alianza Editorial.

"Cedillo, G.; Lizárraga, G y Martner, C. (2019). Medición de la fluidez en corredores de transporte de carga intermodal. Publicación Técnica 544. Querétaro: Instituto Mexicano del Transporte. https://imt.mx/archivos/Publicaciones/ PublicacionTecnica/pt544.pdf

»CEPAL (2016). Panorama de la Inserción Internacional de América Latina y el Caribe 2016: la región frente a las tensiones de la globalización. División de Comercio Internacional e Integración de la Comisión Económica para América Latina y el Caribe-ONU. Santiago: CEPAL. https://www.cepal.org/sites/default/ files/publication/files/40744/S1601274_es.pdf

" CEPAL (2019). Balance Preliminar de las Economías de América Latina y el Caribe 2019. Comisión Económica para América Latina y el Caribe-ONU. Santiago: CEPAL. https://www.cepal.org/es/publicaciones/4500o-balance-preliminareconomias-america-latina-caribe-2019

»Dollfus, O. (2007). La mondialisation. Paris: Presses de Sciences Po.

"Fernández, J. (2018) La hiperglobalización y su impacto. España: Cuadernos de Estrategia $N^{\circ}$ 199:83-118. https://dialnet.unirioja.es/servlet/ articulo? codigo $=6831584$

" Martner, C. (2008). Transporte Multimodal y Globalización en México. México, D.F: Trillas.

» Martner, C. (2010). Puertos, espacio y globalización: el desarrollo de Hubs en México. Convergencia, 17(52), 319-360.

》http://www.scielo.org.mx/scielo.php?script=sci_arttext\&pid=S1405$14352010000100013 \& \operatorname{lng}=e s \& n r m=i s 0$

" Martner, C. (2020). Globalización, conectividad interespacial y articulación territorial de los puertos mexicanos. Revista EURE - Revista de Estudios UrbanoRegionales, 46(139): 233-257. https://www.eure.cl/index.php/eure/article/ view/3176/1305

»Martner, C y A. Pérez (2020). Evolución de la carga por los puertos mexicanos: Entre el neoproteccionismo comercial y el Covid-19. Primera Parte. Gaceta AMANAC, 2 (16): 6-8. https://issuu.com/amanac/docs/gaceta_amanac_ abril_2020

"Miller, G (2020). Container ships suffer record delays as demand spikes. American Shipper:FreightWaves. https://www.freightwaves.com/news/containerships-suffer-record-delays-as-demandspikes?utm_content $=149529440 \& u t m$ medium =social\&utm_source $=$ twitter\&hss_channel $=t w-31128798$

" Miller, G (202ob). Q\&A: COVID, the rise of China, and the future of ocean shipping. American Shipper: FreightWaves. https://www.freightwaves.com/news/ qa-Covid-the-rise-of-china-and-the-future-of-ocean-shipping 
" ONU (2020) Situación y Perspectivas de la Economía Mundial 2020: Resumen ejecutivo. Department of Economic and Social Affairs Economic Analysis. Geneve: United Nations. https://www.un.org/development/desa/dpad/publication/ situacion-y-perspectivas-de-la-economia-mundial-2020-resumen-ejecutivo/

"Santos, M. (2000). La Naturaleza del Espacio. Barcelona: Ariel Geografía.

"Sassen, S. (2003). Localizando ciudades en circuitos globales. Revista EURE, 29(88): 5-27. http://dx.doi.org/10.4067/So250-71612003008800001

»Straight, B. (2021). COVID broke Peloton's supply chain - can \$100M fix it? Modern Shipper: FreightWaves. https://www.freightwaves.com/news/Covidbroke-pelotons-supply-chain-can-10om-fix-it? $p=319051$

» UNCTAD (2020). COVID-19 and Maritime Transport: Impact and responses. Geneve: United Nations Conference on Trade and Development. https://unctad. org/system/files/official-document/dtltlbinf2O20d1_en.pdf

» UNCTAD (2021). Shipping during COVID-19: Why container freight rates have surged. Policy Brief $\mathrm{N}^{\circ} 84$, April 2021. United Nations Conference On Trade And Development. https://unctad.org/system/files/official-document/ presspb2021d2_en.pdf

» Think Goblal Logistic (2021) Record Lows for Global Shipping Schedule Reliability. Sidney: TGL Team. https://tgl.co/record-lows-for-global-shippingschedule-reliability/

»Veltz, P. (2006). Entreprises et territoires, entre fragmentation et intégration, in E. Heurgon (dir) Le développement durable. Paris: Editions de l'Aube.

» Veltz, P. (2014) Mondialisation, villes et territoires: Une économie d'archipel, ze éd. PUF, Paris: Quadrige

»Villahermosa, L. (2021). La fiebre en el precio de los fletes marítimos asfixia al comercio latinoamericano. América Economía. https://www.americaeconomia. $\mathrm{com} /$ negocios-industrias/la-fiebre-en-el-precio-de-los-fletes-maritimosasfixia-al-comercio

"Wallerstein, I. (1996). Después del Liberalismo. México, DF: Siglo XXI Editores.

»Wallerstein, I. (2016). El mundo está desencajado. Interpretaciones históricomundiales de las continuas polarizaciones, 1500-2000. Ciudad de México: Siglo XXI editores.

» Goodman,P; A. Stevenson; N. Chokshi y M.Corkery (2021/03/07). 'I've Never Seen Anything Like This': Chaos Strikes Global Shipping. New York Times. Recuperado de https://www.nytimes.com/2021/03/o6/business/globalshipping.html

»Steven, A. y Phillip, B. (2021/06/23). The State of Globalization in 2021. Harvard Business Review. Recuperado de https://hbr.org/2021/o3/the-state-ofglobalization-in-2021 (18/03/2021).

\section{Carlos Daniel Martner Peyrelongue / martner@imt.mx}

Doctor en Ciencias Sociales con área de especialización en Sociedad y Territorio por la Universidad Autónoma Metropolitana. Coordinador de Transporte Integrado y Logística del Instituto Mexicano del Transporte. 